\title{
Разработка алгоритмов управления процессами охлаждения продуктов в туннельных камерах
}

\section{С. М. Дубна, А. А. Гурский, А. Е. Гончаренко, Н. А. Пантелюк}

Учебно-научный институт механики, автоматизации и робототехники им. П.Н. Платонова Одесской национальной академии пищевых технологий, 65039, ул. Канатная, 112, г. Одесса, Украина

В статье рассматриваются системы управления процессами охлаждения продуктов в туннельных камерах. Представляется разработанная лабораторная холодильная установка с туннельной камерой как физическая модель для экспериментальных исследований рассматриваемых систем управления. Реализуются модели систем управления в среде MATLAB\imulink. Представляются законы управления различных систем. Выполняется сравнительный анализ результатов моделирования систем управления для дальнейшей проверки принципиальной пригодности их на базе лабораторной холодильной установки. В заключительной части работы делается вывод об актуальности применения системы управления, функционирующей по принципу согласованного регулирования и координирующего управления.

Ключевые слова: Туннельная камера; Холодильная установка; Согласованное регулирование; Координирующее управление; Энергоэффективность; Динамическая точность

\section{Розробка алгоритмів управління процесами охолодження продуктів у тунельних камерах}

\section{С.М. Дубна, О.О. Гурський, О.С. Гончаренко, М.О. Пантелюк}

Навчально-науковий інститут механіки, автоматизації і робототехніки ім. П.М. Платонова Одеської національної академії харчових технологій, 65039, вул. Канатна, 112, м. Одеса, Україна

У статті розглядаються системи управління процесами охолодження продуктів у тунельних камерах. Надається розроблена лабораторна холодильна установка з тунельною камерою як фізична модель для експериментальних досліджень розглянутих систем управління. Реалізуються моделі систем управління в середовищі MATLAB\imulink. Виконується порівняльний аналіз результатів моделювання систем управління для подальшої перевірки приничиової придатності їх на базі лабораторної холодильної установки. Надаються закони управління різноманітних систем. У заключній частині роботи робиться висновок про актуальність застосування системи управління, функціонуючої за принципом погодженого регулювання $і$ координуючого управління.

Ключові слова: Тунельна камера; Холодильна установка; Погоджене регулювання; Координуюче управління; Енергоефективність; Динамічна точність

(C) The Author(s) 2017. This article is an open access publication

This work is licensed under the Creative Commons Attribution 4.0 International License (CC BY) http://creativecommons.org/licenses/by/4.0/

\section{1. Введение}

Холодильные туннели или туннельные камеры имеют широкое применение там, где необходимо получить качественный продукт при правильном охлаждении. Качество охлаждения продукта обеспечивается высокой точностью поддержания температуры независимо от возмущающих факторов. При этом повышение точности поддержания температуры играет важное значение как при охлаждении определенных продуктов, так и при обеспечении энергоэффективного функционирования холодильной установки.
Очевидно, что точность поддержания температуры осуществляется посредством регулирования производительности холодильного компрессора, однако следует учесть еще множество различных факторов. Один из таких факторов - это температура продукта на входе в камеру, скорость движения транспортерной ленты или сетки в туннельной камере и соответствующая скорость поступления продукта в камеру, а также одни из важных факторов - это производительность нагнетателей холодного воздуха в туннельную камеру. В данном случае можно выделить различные управляющие воздействия, например, по скорости движения ленты транспортера и загрузки 
продукта, а также по производительности нагнетателей воздуха.

Применение различных управляющих воздействий для обеспечения точности поддержания температуры дает возможность рассмотреть различные структурные схемы САУ, однако для выделения наиболее удачного варианта схемы САУ требуется проведение различных экспериментов и сравнительных анализов функционирования установки.

Один из действенных методов повышения динамической точности САУ связан с обеспечением инвариантности некоторых переменных в системе или с обеспечением динамической развязки (автономности) каналов регулирования. Однако, как отмечалось в некоторых работах, системы, построенные на принципах автономности, не всегда могут показать желаемые результаты. Например, «системы управления живыми организмами, которые совершенствовались в течение целых геологических эпох, построены не на принципе автономности, a, наоборот, на принципе исключительно тесного взаимодействия, что обеспечивает высокие динамические качества» $[1,2]$.

При особой важности поддержания температуры с определенной точностью актуально рассмотреть принцип согласованного регулирования или координирующего управления, для обеспечения согласованного изменения скорости движения транспортерной ленты и производительности нагнетателей холодного воздуха в камеру при процессе регулирования температуры [3, 4].

Таким образом, в настоящей работе рассматриваются модели различных систем управления, в том числе модель системы, отражающей принцип согласованного регулирования, для дальнейшего применения этих систем на базе имеющейся физической модели - холодильной лабораторной установки с туннельной камерой

\section{Цель и задача настоящей работы}

Целью работы является повышение динамической точности САУ и энергоэффективности функционирования холодильных установок с туннельными камерами.

Для достижения поставленной цели необходимо реализовать синтез различных систем управления процессами охлаждения продуктов в туннельных камерах, a также проверить принципиальную пригодность данных синтезированных систем управления на базе холодильной лабораторной установки с туннельной камерой.

\section{2. Основная часть}

Технологические особенности разработан-ной лабораторной установки

Лабораторная холодильная установка, общий вид которой представлен на рисунке 1, разработана в лаборатории учебно-научного комплекса «Автоматизация холодильных процессов» кафедры АТП и РС ОНАПТ, и предназначена для исследования энергоэффективных систем управления процессами охлаждения. Данная установка, по сути, является физической моделью установки с туннельной морозильной камерой, предназначенной для замораживания полуфабрикатов, мелкоштучного или измельченного плодовоовощного сырья, субпродуктов, мяса, рыбы и т.д. В лабораторной установке в качестве охлаждаемого сырья выступает вода, которая подается в стаканы при прохождении их на транспортере через инфракрасный барьер.

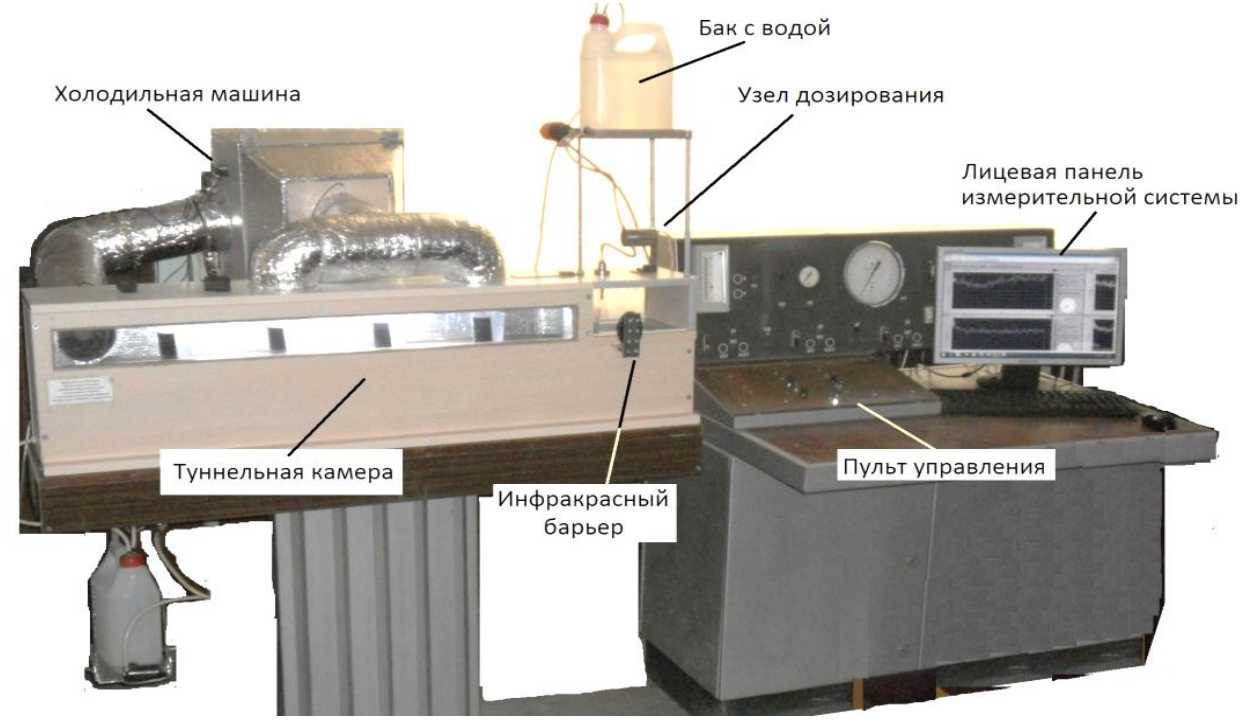

Рисунок 1 - Общий вид лабораторной холодильной установки с туннельной камерой

Измерительная система, реализованная на базе среды LabVIEW, отражает временные диаграммы изменения температур, полученных от 6-ти датчиков, установленных в различных точках холодильной установки. Управление установкой реализуется в ручном режиме, а также лабораторная установка реализуется под автоматическим управлением среды
LabVIEW, в которой были отработаны различные алгоритмы управления. При этом связь с объектом, как показано на структурно-технологической схеме (рисунок 2), реализуется на базе микроконтроллера, соединенного с компьютером по USB, и модуля управления моторами Motor Driver L298N. 


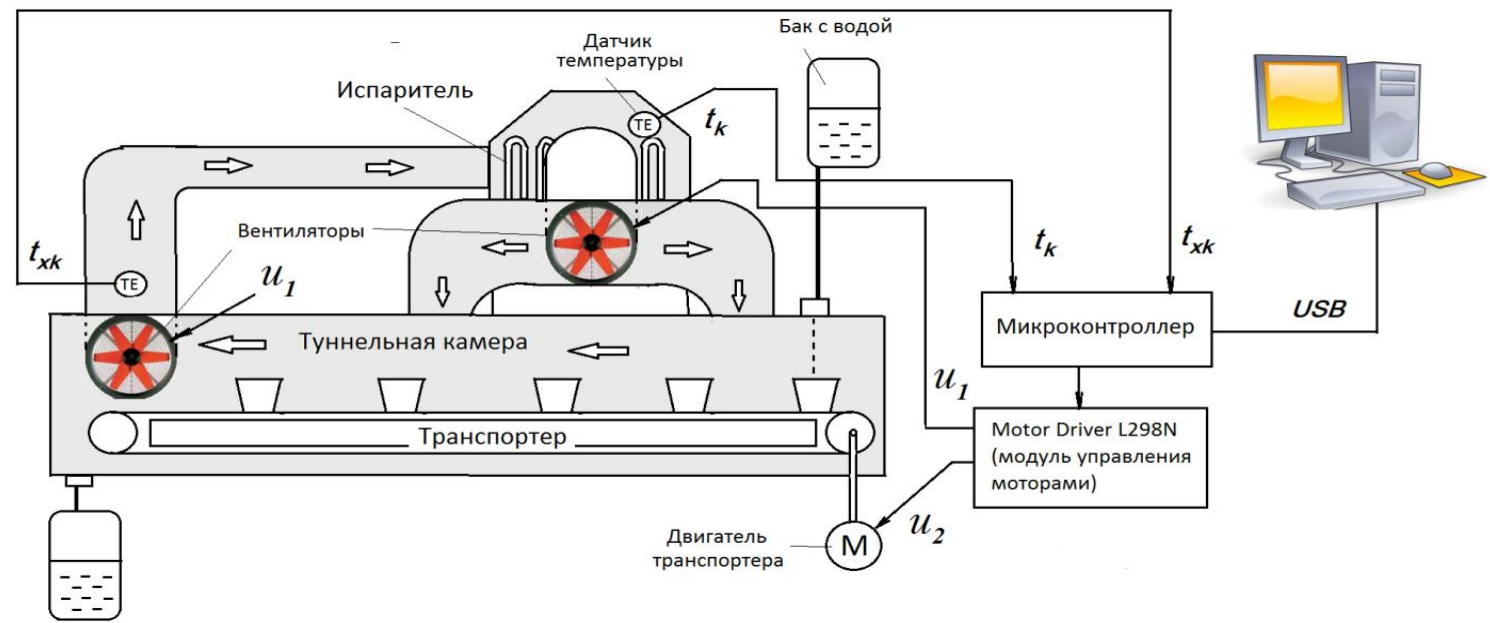

Рисунок 2 - Структурно-технологическая схема лабораторной холодильной установки с туннельной камерой

Синтез систем автоматического регулирования

Для синтеза систем автоматического управления были проведены активные эксперименты для определения динамической модели лабораторной установки. В результате идентификации динамических и статических параметров модели было установлено, что уравнения динамики объекта можно записать в следующем виде:

$$
\begin{aligned}
& t_{k}(p)=W_{11}(p) \cdot u_{1}(p)+W_{12}(p) \cdot u_{2}(p)+t_{k 0} \\
& t_{k x}(p)=W_{21}(p) \cdot u_{1}(p)+W_{22}(p) \cdot u_{2}(p)+t_{k x 0},
\end{aligned}
$$

где $t_{k}-$ значение температуры на испарителе; $t_{x k}-$ значение температуры в конце туннельной камеры $t_{k 0}=$ $-10^{\circ} \mathrm{C} ; t_{x k 0}=-4^{\circ} \mathrm{C}-$ значение температур на испарителе и в конце туннельной камеры при выключенных вентиляторах и без подачи в камеру охлаждаемого продукта;

$W_{11}(p), \quad W_{12}(p), \quad W_{21}(p), \quad W_{22}(p)$ - передаточные функции по соответствующим каналам регулирования.

В процессе идентификации были определены следующие передаточные функции:

$$
\begin{aligned}
& W_{11}(p)=\frac{0,02 \cdot \exp (-3 p)}{(15 \cdot p+1)^{2}} ; \\
& W_{12}(p)=\frac{0,02 \cdot \exp (-6 p)}{(90 \cdot p+1)^{2}} ; \\
& W_{21}(p)=\frac{0,04 \cdot \exp (-7 p)}{(10 \cdot p+1) \cdot(110 \cdot p+1)} ; \\
& W_{22}(p)=\frac{0,07 \cdot \exp (-7 p)}{(90 \cdot p+1)^{2}} .
\end{aligned}
$$

Таким образом, в настоящем случае представлен объект достаточно распространенного вида - линейный многоканальный объект, число выходных переменных которого равно числу управляющих воздействий.

При необходимости стабилизации температуры на испарителе, соответствующей температуре кипения, и температуры в конце туннельной камеры, реализуется типовая САУ, в структуру которой включены стандартные ПИ-регуляторы. Законы функционирования таких регуляторов имеют вид:

$$
\begin{aligned}
& u_{1}(p)=W_{P I}(p) \cdot e_{1}(p) \\
& e_{1}(p)=t_{k z}-t_{k}(p) ; \\
& p=d / d t \\
& u_{2}(p)=W_{P I}(p) \cdot e_{2}(p) \\
& e_{2}(p)=t_{x k z}-t_{x k}(p)
\end{aligned}
$$

где $W_{P I}(p)=k_{1}+k_{2} / p ; \quad k_{1}$ и $k_{2}-$ коэффициенты настройки; $t_{k z}, t_{x k z}-$ заданные значения температуры на испарителе и температуры в конце туннельной камеры.

В процессе моделирования систем были рассмотрены различные пути повышения динамической точности САУ и улучшения качества регулирования. Один из распространенных вариантов улучшения качества регулирования реализуется за счет обеспечения автономности контуров регулирования или динамической развязки каналов регулирования. В данном случае закон управления будет иметь следующий вид:

$$
\begin{aligned}
& u_{1}(p)=W_{P I 1}(p) \cdot e_{1}(p)+W_{k 1}(p) \cdot u_{2}(p) \\
& u_{2}(p)=W_{P I 2}(p) \cdot e_{2}(p)+W_{k 2}(p) \cdot u_{1}(p)
\end{aligned}
$$

где $W_{k 1}(p), W_{k 2}(p)$ - передаточные функции звеньев в перекрестных связях, обеспечивающих динамическую развязку каналов регулирования.

Очевидно, что не ожидается явное улучшение качества регулирования при включении корректирующих звеньев с передаточными функциями $W_{\mathrm{k} 1}(p), W_{\mathrm{k} 2}(p)$ так как канал регулирования $u_{l}-t_{k}$ существенно менее инерционный, чем перекрестный канал $u_{2}-t_{x k}$. Однако во многих случаях достижение автономности каналов регулирования позволяет улучшить динамическую точность САУ.

Совершено противоположным направлением автономности контуров регулирования является оптимальная динамическая связность каналов регулирования. В данном случае цель управления 
может быть представлена в виде уравнений, связывающих значения выходных величин объекта: $y_{i}\left(x_{1} \ldots x_{n}\right)=0$, где $x_{n}$ - выходная переменная объекта управления. При этом реализуется согласование переходных процессов в динамической системе. Структурная схема модели системы согласованного регулирования представлена на рисунке 3 средствами среды MATLAB $\backslash$ Simulink.

Закон управления при согласовании переходных процессов двухуровневый, имеет следующий вид:

$$
u=\bar{u}_{p}+\bar{u}_{q}
$$

где

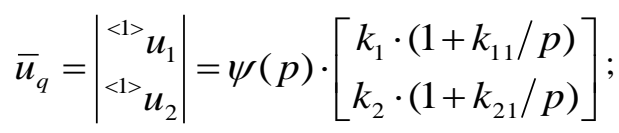

$\bar{u}_{p}=\left|\begin{array}{c}0 \\ <2> \\ u_{2}\end{array}\right|=e_{2}(p) \cdot\left[\begin{array}{c}0 \\ k_{3} \cdot\left(1+k_{31} / p\right)\end{array}\right] ; \quad p=d / d t ;$

$\psi(p)=A^{T} \cdot X-b ;$

$A^{T}=\left[\begin{array}{ll}3 & -1\end{array}\right]-$ вектор коэффициентов соотношения;

$X=\left[\begin{array}{ll}t_{x k} & t_{k}\end{array}\right]^{T}$ - вектор регулируемых переменных; $k_{l}$, $k_{2}, k_{3}, k_{11}, k_{21}, k_{31}$ - параметры настройки.

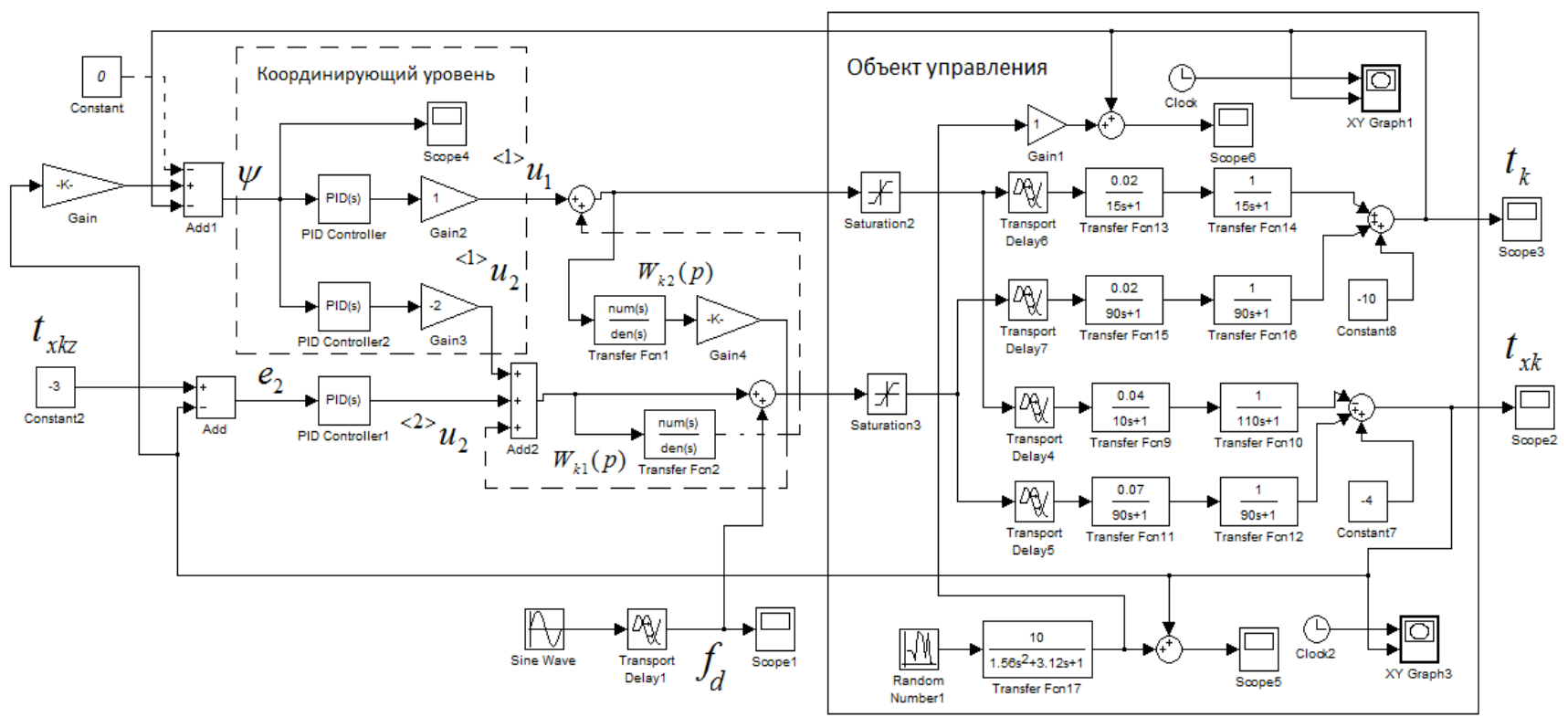

Рисунок 3 - Структурная схема модели САУ установки с туннельной камерой

При этом 1-й уровень $\left(\bar{u}_{q}\right)$ связан с регулированием соотношения выходных переменных, а 2-й уровень $\left(\bar{u}_{p}\right)$ - со стабилизацией выходных переменных на заданных значениях.

\section{Анализ результатов моделирования}

В результате моделирования были получены переходные процессы по температуре на испарителе и по температуре в конце туннельной камеры при различных САУ. Из графиков переходных процессов, представленных на рисунках $4-6$, видно, что автономность контуров регулирования не приводит к существенному улучшению качества регулирования, однако совершено иной результат получен, когда в системе управления регулируемые температуры были связаны соотношением. Как видно из рисунка 4, существенно уменьшилось перерегулирование и максимальное динамическое отклонение по температуре в конце туннельной камеры при согласованном регулировании, в отличие от типовой САУ, хотя параметры настройки регуляторов температур не менялись.

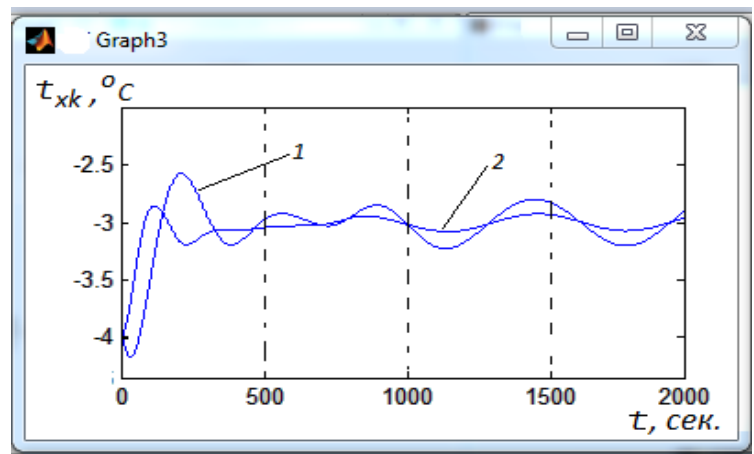

$4 \mathrm{a}$

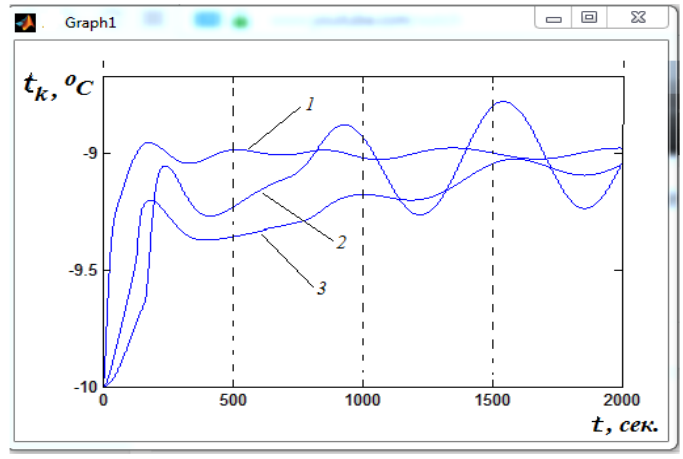

46

Рисунок 4 - Переходные характеристики по температуре на испарителе (рисунок 4а) и по температуре в конце туннельной камеры (рисунок 4б), кривая 1 - изменение температуры во времени при типовой САУ без динамической развязки каналов регулирования; кривые 2 и 3 - изменение температур во времени при различных значениях параметров настройки координирующего уровня системы согласованного регулирования 


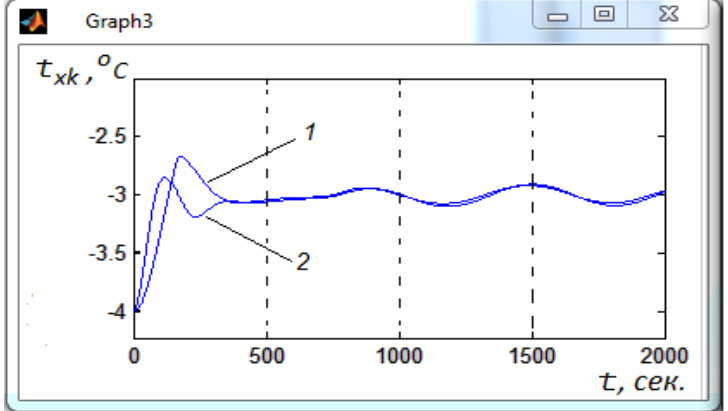

Рисунок 5 - Переходные характеристики по температуре в конце туннельной камеры при различных значениях параметров настройки координирующего уровня

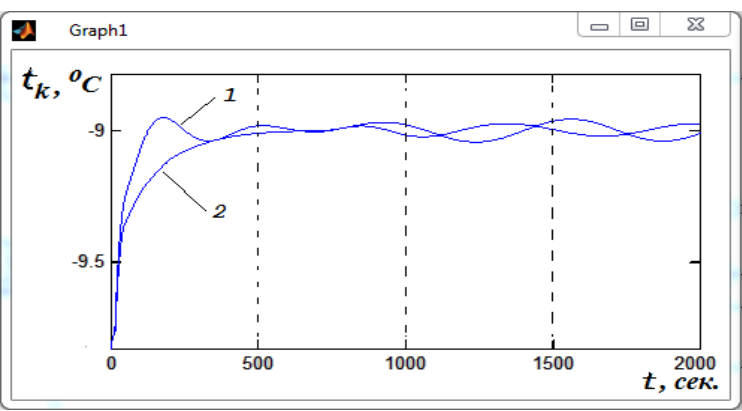

Рисунок 6 - Переходные характеристики по температуре на испарителе, кривая 1 - при типовой САУ; кривая 2 - при САУ с автономными контурами регулирования

\section{3. Выводы}

Проведенный сравнительный анализ различных САУ процессами охлаждения продуктов в туннельных камерах показал существенное положительное изменение качества регулирования температуры при применении в системе принципов согласованного регулирования.

Разработанные модели САУ позволяют в дальнейшем реализовать соответствующие алгоритмы управления на базе имеющейся физической модели холодильной установки с туннельной камерой. Это дает возможность в дальнейшем провести определенные эксперименты в области повышения энергоэффективности функционирования холодильных установок.

\section{Литература}

1. Морозовский В.T. Многосвязные системы автоматического управления / В. Т. Морозовский. - М. : Энергия, 1970. - 288 с.

2. Соболев О.С. Методы исследования линейных многосвязных систем.- М. : Энергоатомиздат, 1985. $120 \mathrm{c}$.

3. Мирошник И.В. Согласованное управление многоканальными системами / И. В. Мирошник. - Л. : Энергоатомиздат. $1990-128 \mathrm{c.}$

4. Бойчук Л.М. Синтез координирующих систем автоматического управления - М.: Изд-во «Энергоатомиздат», 1991. - $160 \mathrm{c.}$

Отримана в редакції 02.08.2017, прийнята до друку 08.09.2017

\section{The working out of algorithms in control of the cooling processes of products in the tunnel cameras}

\section{S. M. Dubna, A. A. Gursky, A. E. Goncharenko, N. A. Panteleiq}

The P.N. Platonov institute of mechanics, automation and robotics. Odessa national academy of food technologies. $1 / 3$ Dvoryanskaya str. Odessa, 65082, Ukraine

The control systems of cooling processes of products in the tunnel chambers is considered in this article. The designed laboratory refrigeration system with the tunnel chamber is represented in this scientific work. This plant is as the physical model for experimental research of the control systems. The laboratory refrigeration plant is controlled by means of the LabVIEW environment. We have used the microcontroller connected to the computer via USB and the module of motor control. The purpose of scientific work the dynamic accuracy increase of the control system and efficiency of the exploitation of refrigeration plant with tunnel chamber. We have fulfilled the active experiments to determine the dynamic and static parameters of the plant as the control object. The models of control systems in the $M A T L A B \backslash$ Simulink environment are realized. The comparative analysis of the results of simulation of control systems is also performed. It is necessary to verify the principal suitability on the basis of a laboratory refrigeration plant. The control of various systems laws are presented in this paper. At the end of this work we have made the conclusion about the relevance of using the control system. This system functions on the principle of the coordinated regulation and the coordinating control.

Keywords: Tunnel chamber; Refrigeration plant; Coordinating control; Energy efficiency

\section{Reference}

1. Morozovskii V.T. (1970) Mnogosvyaznie sistemi avtomaticheskogo upravleniya. Moscow, Energia, 288 p. 2. Sobolev O.S. (1985) Metodi issledovania lineinih mnogosvyaznih sistem. Moscow, Energoatomizdat, 120 p.

3. Miroshnik I.V. (1990) Soglasovannoe upravlenie mnogokanalnimi sistemami. Leningrad, Energoatomizdat, $128 \mathrm{p}$.

4. Boichuk L.M. (1991) Sintez koordiniruyuschih sistem avtomaticheskogo upravleniya. Moscow, Energoatomizdat, $160 \mathrm{p}$.

Received 02 August 2017 Approved 08 September 2017 Available in Internet 30 October 2017 